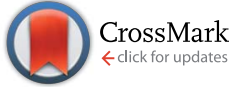

Cite this: RSC Adv., 2017, 7, 14438

Received 7th December 2016 Accepted 24th February 2017

DOI: 10.1039/c6ra27893k

rsc.li/rsc-advances

\section{Blocking celiac antigenicity of the glutamine-rich gliadin 33-mer peptide by microbial transglutaminase $\uparrow$}

\author{
Lin Zhou, ${ }^{\text {abc }}$ Yujie Wu, ${ }^{\text {abc }}$ Youfei Cheng, ${ }^{\text {abc }}$ Jie Wang, ${ }^{b}$ Jun Lu, ${ }^{\text {abc }}$ Jinyan Gao, ${ }^{\text {ab }}$ \\ Juanli Yuan ${ }^{\text {ad }}$ and Hongbing Chen*ab
}

Celiac disease (CD) is a T cell-mediated enteropathy of the small intestine and caused by the ingestion of wheat gluten and related prolamins in barley and rye. However, there is no effective therapy to alleviate symptoms of celiac disease except for a life-long gluten-free diet. Recent studies showed that modification by microbial transglutaminase ( $\mathrm{mTG}$ ) could reduce the gliadin-specific immune response. In the present study, different acyl-acceptor substrates in combination with mTG were used to modify the model 33-mer peptide (LQLQPFPQPQLPYPQPQLPYPQPQLPYPQPQPF), which is a particular celiac toxic $\alpha$-gliadin peptide. RP-HPLC and LC-ESI-MS were performed to test the extent of the modifications. R5 ELISA and G12 ELISA were used to analyze the antigenicity of the modified peptide. The shifts of retention time and molecular weight showed great modification of 33-mer peptide after $2 \mathrm{~h}$ of incubation with mTG. When acyl-acceptor substrates were crosslinked with 33-mer peptide, the antigenicity of modified peptide forms was decreased compared to its initial level. In summary, it is demonstrated that mTG is active on a variety of chemically acyl-acceptor substrates. Transamidation by mTG with an appropriate amine donor can be used to block the antigenicity of gliadin peptide related to celiac disease. These findings highlight a potential strategy to prevent cereal toxicity in celiac disease.

\section{Introduction}

Celiac disease (CD) is an intestinal disorder developed in genetically susceptible individuals and caused by the ingestion of wheat gluten and related prolamins present in barley and rye. $^{1} \mathrm{CD}$ is strongly related to the HLA locus, specifically HLA-DQ2 (A1*0501-B1*0201) and/or HLA-DQ8 (A1*0301$\mathrm{B} 1 * 0302) .{ }^{2}$ The gluten epitopes can induce $\mathrm{CD}$ after deamidation by tissue transglutaminase (TG2), which is a crucial step in CD pathogenesis., ${ }^{3,4}$ The deamidated gluten epitopes have higher affinity to bind to HLA-DQ2 and HLA-DQ8 molecules, leading to stronger T-cell responses. ${ }^{5}$ Furthermore, the primary toxic components of wheat gluten are gliadins, which are glutamine-rich and proline-rich proteins with resistance to digestion. ${ }^{6,7}$ Moreover, there exists a particular toxic $\alpha$-gliadin peptide, LQLQPFPQPQLPYPQPQLPYPQPQLPYPQPQPF, which

${ }^{a}$ State Key Laboratory of Food Science and Technology, Nanchang University, 235 Nanjing Dong Road, Nanchang 330047, China. E-mail: chenhongbing@ncu.edu.cn; Fax: +8679188333708; Tel: +8679188334552

${ }^{b}$ School of Food Science and Technology, Nanchang University, Nanchang 330031, China

'Sino-German Joint Research Institute, Nanchang University, Nanchang 330047, China ${ }^{d}$ College of Pharmaceutical Sciences, Nanchang University, Nanchang 330031, China $\uparrow$ Electronic supplementary information (ESI) available. See DOI: $10.1039 / \mathrm{c} 6 \mathrm{ra} 27893 \mathrm{k}$ consists of 33 amino acids including 10 glutamine residues. The 33-mer peptide is naturally formed by digestion with gastric and pancreatic enzymes and is resistant to further degradation by enzymes of the brush-border membrane (BBM). ${ }^{6}$ The peptide contains three overlapping immunodominant epitopes (Fig. 1A), PFPQPQLYP (one copy), PYPQPQLPY (two copies) and PQPQLPYPQ (three copies) in that their deamidated forms can stimulate T cells to induce adaptive immunity. ${ }^{6,8}$ However, there is no effective therapy to treat $\mathrm{CD}$ except for a life-long glutenfree diet, which is arduous to persist with because of high prices, limited variety and poor sensory properties. ${ }^{9-11}$

In this sense, enzymatic modification of gluten has been utilized to decrease the CD-specific immunological activities without affecting other principal properties. Gluten proteins were modified by binding methionine to the amino lateral groups of glutamine residues with chymotrypsin, which reduced the induction of celiac IgA. ${ }^{\mathbf{1 2}}$ Furthermore, food-grade microbial transglutaminase (mTG) has been used to reduce the immunological activity of gluten proteins, which typically catalyses $\mathrm{pH}$ dependent transamidation to form an $\varepsilon$-( $\gamma$-glutamyl)-lysine bond between glutamine residues (acyl-donor substrate) and lysine residues (acyl-acceptor substrate). ${ }^{\mathbf{1 3}}$ Detoxification was attributed to the selective transamidation of glutamine residues, which blocked the deamidation process by mTG and tTG, which is critical for the development of CD. Although mTG can also catalyse a hydrolysis reaction between glutamine residues and 
A

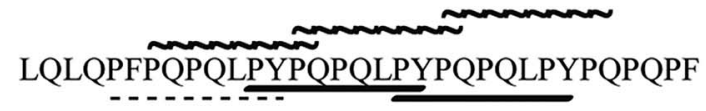

B LQLQPFPQPQLPYPQPQLPYPQPQLPYPQPQPF

C

LYS

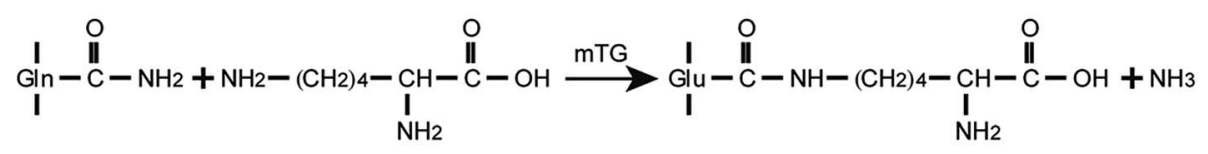

LME

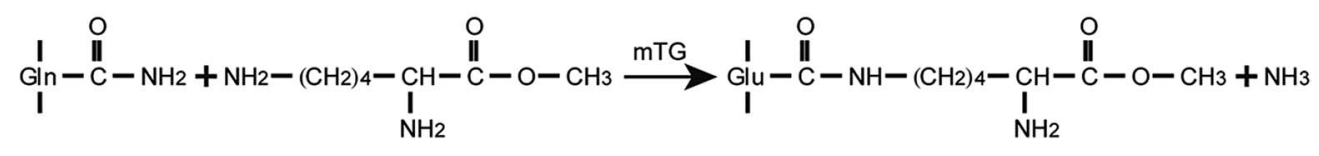

GEE

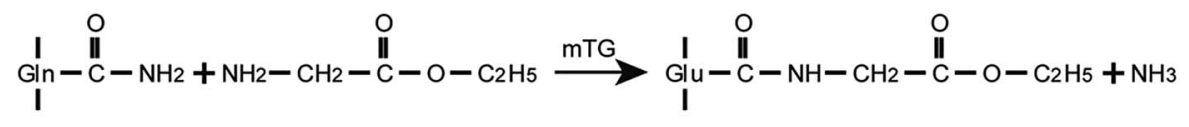

6-AA

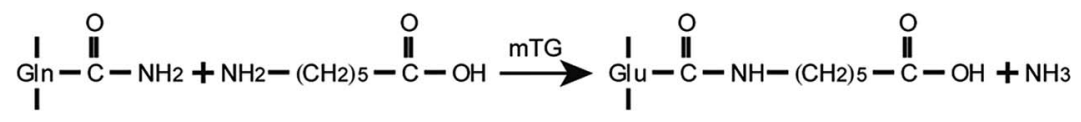

HA

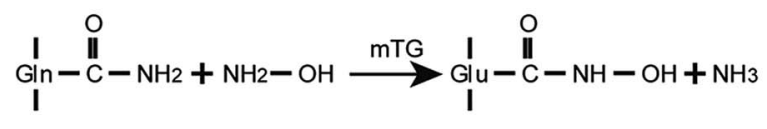

DA

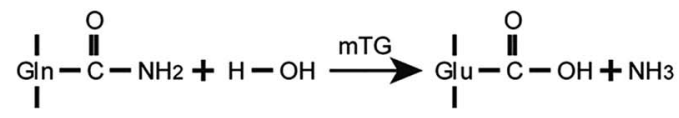

Fig. 1 Structure of the 33-mer peptide, detection mechanism of ELISA and catalytic mechanism of mTG. (A) Structure of the 33-mer peptide contains three overlapping immunodominant epitopes, PFPQPQLYP (one copy, shown in dash line), PYPQPQLPY (two copies, shown in bold line) and PQPQLPYPQ (three copies, shown in wavy line); (B) the detection mechanism of R5 ELISA and G12 ELISA. LQPFP (one copy, shown in bold line) in the 33-mer peptide can be recognized by R5 mAb, whereas the sequence of QLPYP (three copies, shown in dash line) remains a weak reactivity to the antibody. QPQLPY (three copies, shown in wavy line) in the 33-mer peptide were specifically recognized for G12 mAb; (C) mechanism of mTG. Glutamine residues (acyl-donor substrates) reacted with LYS, LME, GEE, 6-AA and HA (acyl-acceptor substrates) in the presence of MTG. Without acyl-acceptor substrates, MTG catalysed the deamidation reaction. mAb: monoclonal antibody; LYS: L-lysine; LME: Llysine methyl ester; GEE: glycine ethyl ester; 6-AA: 6-aminocaproic acid; HA: hydroxylamine; DA: deamidation.

$\mathrm{H}_{2} \mathrm{O}$, it has a lower deamidation preference than human tissue transglutaminase (tTG). ${ }^{14}$ It was found that the reactivity between gluten proteins and $\mathrm{R} 5 \mathrm{mAb}$ was reduced by $42 \%$ using R5 ELISA when gluten proteins were modified by $\mathrm{mTG}$ and lysine. ${ }^{15}$ Moreover, previous studies show that the transamidation by mTG/tTG with appropriate acyl-acceptor substrates (lysine or lysine methyl ester) can be used to prevent the immunological activity of gluten proteins, while as it was not sufficient in abolishing the gluten activity. ${ }^{16-19}$ In addition, recent study highlighted the potential of mTG transamidation to block nonimmune mechanisms related to gluten toxicity. ${ }^{20}$
On this basis, it is of interest to study other compounds that might substitute for lysine as acyl-acceptor substrates with the aim of diversifying biocatalytic applications of mTG in the modification of gluten and the breadth of utility as a green biocatalyst in the food industry. In this experiment, it was the aim to investigate the transamidation of the 33-mer peptide using mTG and different acyl-acceptor substrates, namely $\mathrm{L}^{-}$ lysine (LYS), L-lysine methyl ester (LME), glycine ethyl ester hydrochloride (GEE), 6-aminocaproic acid (6-AA) and hydroxylamine (HA), which have different molecular weights (Fig. 1C). Furthermore, in vitro digestions of the 33-mer peptide and its 
modified forms were executed by trypsin and $\alpha$-chymotrypsin. Reversed-phase high-performance liquid chromatography (RPHPLC) and liquid chromatography-electrospray ionizationmass spectrometry (LC-ESI-MS) were performed to test the extent of modifications. R5 ELISA and G12 ELISA were used to analyze the possible changes of antigenicity after the transamidation of mTG and digestion of enzyme. R5 ELISA is suggested by Codex Alimentarius for detection and quantification of gluten in food and G12 ELISA is specific for the detection of the 33-mer peptide.

Our hypothesis was that the acyl-acceptor substrates in the experiment can be crosslinked efficiently to glutamine residues of toxic epitopes, resulting in a low level of CD toxic epitopes. Moreover, the isopeptide bonds of modified 33-mer peptide were resistant to the digestion of digestive enzymes, which indicated that all of the transamidated forms would be stable.

\section{Materials and methods}

\subsection{Synthetic peptides and chemicals}

The 33-mer peptide was synthesized by GL Biochem Ltd (Shanghai, China). The purity $(\geq 95 \%)$ and structure were assessed by reversed-phase high performance liquid chromatography (RP-HPLC) and liquid chromatography-electrospray ionization-mass spectrometry (LC-ESI-MS). MTG was donated by Jiangsu Yiming Biological Products Co., Ltd. (Jiangsu, China) with a declared activity of 1000 units per g. LYS (>98\%), GEE (>98\%) and 6-AA (>98.5\%) were bought from Sangon Biotech (Shanghai, China). LME (98\%) and HA (98\%) were from Aladdin (Shanghai, China) and Sigma-Aldrich, respectively. Pepsin $(\geq 2500$ units per $\mathrm{mg}$ protein), trypsin $(\geq 7500$ BAEE units per $\mathrm{mg}$ ), $\alpha$-chymotrypsin (Type II, lyophilized powder, $\geq 40$ units per $\mathrm{mg}$ protein) and bile salt (sodium glycodeoxycholate and sodium taurocholate) were purchased from Sigma-Aldrich. All reagents used in RP-HPLC analysis were of HPLC grade. Other chemicals were of analytical grade.

\subsection{Determination of mTG activity}

An amount of $0.5 \mathrm{~g}$ enzyme powder was dissolved in $25 \mathrm{~mL}$ $0.01 \mathrm{~mol} \mathrm{~L}^{-1}$ phosphate buffer, and the mixture was centrifuged at $5000 \mathrm{~g}$ to remove precipitate. ${ }^{21}$ The supernatant was dialyzed with a molecular weight cut-off of $3500 \mathrm{Da}$ to remove other components. The resulting enzyme solution was stored at $4{ }^{\circ} \mathrm{C}$, and directly used for subsequent reaction. Enzyme activity was determined by a colorimetric procedure using Z-Gln-Gly as the substrate. $^{22}$ Z-Gln-Gly reacted with hydroxylamine hydrochloride in the presence of $\mathrm{mTG}$ and then the products were combined with ferric ion in acidic conditions. The enzymatic activity was evaluated by UV absorbance at $525 \mathrm{~nm}$. Meanwhile, the calibration curve is established on the basis of the concentration of L-glutamic acid $\gamma$-monohydroxamate as standard. One enzyme unit (U) is defined as the formation of $1 \mu \mathrm{mol}$ L-glutamic acid $\gamma$-monohydroxamate per min at $37^{\circ} \mathrm{C}$.

Based on the standard curve, the calculated enzyme activity was calculated as about $10 \mathrm{U} \mathrm{mL}^{-1}$.

\subsection{Modification by mTG}

In the experiments, five transamidation mixtures and one deamidation mixture were performed. Regarding the transamidation, each acyl-acceptor substrate in Table 1 was used in five different transamidation mixtures, respectively. The reaction solution contained $1 \mathrm{mg}$ of 33-mer peptide, $0.5 \mathrm{U}$ mTG and $0.05 \mathrm{mmol}$ acyl-acceptor substrate in a final volume of $1 \mathrm{~mL}$, as well as the control groups were prepared using $1 \mathrm{mg}$ of 33-mer peptide, $0.05 \mathrm{mmol}$ acyl-acceptor substrate and inactivated mTG. Regarding the deamidation reaction, the mixture was performed with $1 \mathrm{mg}$ of 33-mer peptide, $0.5 \mathrm{U}$ mTG and without the acylacceptor substrates. The assay was carried out at $50{ }^{\circ} \mathrm{C}$ for $2 \mathrm{~h}$, followed by stopping the enzymatic reaction by heating at $85{ }^{\circ} \mathrm{C}$ for $10 \mathrm{~min}$.

\subsection{RP-HPLC analysis and LC-ESI-MS analysis}

2.4.1 RP-HPLC analysis. A total of $1 \mathrm{~mL}$ aliquots of the sample solution was filtered through a $0.22 \mu \mathrm{m}$ MCE membrane (SHIMADZU-GL SCIENCES, Shanghai, China), followed by analyzing $10 \mu \mathrm{L}$ using RP-HPLC using a C8 column (4.6 mm i.d. $\times 250 \mathrm{~mm}, 5 \mu \mathrm{m}$, Inertsil WP300; GL Sciences, Japan). The eluents used were as follows: (A) $0.1 \%$ trifluoroacetic acid (TFA) in water and (B) $0.1 \%$ TFA in acetonitrile. First, the column was equilibrated in $25 \%$ solvent $\mathrm{B}$. Then a $30 \mathrm{~min}$ gradient of $25-$ $50 \%$ buffer B was used. The flow rate was $0.8 \mathrm{~mL} \mathrm{~min}^{-1}$ on a LC20AT model system (SHIMADZU, Japan) and the column temperature was $25^{\circ} \mathrm{C}$. The samples were detected at $220 \mathrm{~nm}$.

2.4.2 LC-ESI-MS analysis. Samples analysis were carried out on LC-ESI-MS (Agilent, USA) in positive-ion reflectron mode. Solvent A was $0.1 \%$ formic acid in water and solvent B was $0.1 \%$ formic acid in acetonitrile. In the first place, samples were separated on a C8 column $(4.6 \mathrm{~mm}$ i.d. $\times 250 \mathrm{~mm}, 5 \mu \mathrm{m}$, Inertsil WP300; GL Sciences, Japan) using a linear gradient of $25-50 \%$ B over $25 \mathrm{~min}$. The samples were operated at $220 \mathrm{~nm}$. Fragmentor and capillary voltage were $135 \mathrm{~V}$ and $4000 \mathrm{~V}$ respectively. The flow rate was split from 200 to $5 \mu \mathrm{L} \mathrm{min}{ }^{-1}$ using a flow splitter. MS mode acquisition was performed over the $m / z$ range $100-2000$.

\subsection{In vitro digestions of the 33-mer peptide and its modified forms}

The in vitro digestions of each peptide were performed according to previous studies with some modifications. ${ }^{23}$ For gastric digestions, porcine pepsin was dissolved in simulated gastric fluid with $35 \mathrm{mM} \mathrm{NaCl}$ at $\mathrm{pH}$ 2.0. Gastric digestions were performed for $60 \mathrm{~min}$ at $37^{\circ} \mathrm{C}$ with the ratio of $1: 50$ (enzyme to peptide, w/w). Aliquots were withdrawn at $60 \mathrm{~min}$. The digestion reactions were stopped by adjusting the $\mathrm{pH}$ to 7.0 with $\mathrm{NaOH}$. Duodenal digestion was conducted using the 60 min gastric digests as the starting material. Moreover, the reaction mixtures were supplemented with bile salt, bis-tris, $\mathrm{CaCl}_{2}$ and enzymes (trypsin and $\alpha$-chymotrypsin). The final composition was $6.15 \mathrm{mM}$ of bile salt, $20.3 \mathrm{mM}$ bis-tris, $7.6 \mathrm{mM} \mathrm{CaCl}_{2}$, trypsin ( $1: 50$, enzyme to protein, w/w) and $\alpha$-chymotrypsin $(1: 200$, enzyme to protein, w/w). The reactions were incubated at $37{ }^{\circ} \mathrm{C}$ 
Table 1 Reactivity of five acyl-acceptor substrates with 33-mer peptide catalysed by $\mathrm{mTG}^{a}$

\begin{tabular}{|c|c|c|c|c|}
\hline $\begin{array}{l}\text { Acyl-acceptor } \\
\text { substrates }\end{array}$ & Structure & MW & $\mathrm{RT}^{b}(\min )$ & Percentage $^{c}(\%)$ \\
\hline $1 \mathrm{LYS}$ & & 146.19 & 14.64 & $90.26 \pm 4.43$ \\
\hline 2 LME & & 160.21 & 15.26 & $47.51 \pm 2.47$ \\
\hline 3 GEE & & 103.12 & 20.10 & $42.12 \pm 0.33$ \\
\hline $5 \mathrm{HA}$ & & 33.02 & 16.31 & $36.51 \pm 0.31$ \\
\hline
\end{tabular}

for $120 \mathrm{~min}$, followed by stopping by heating at $100{ }^{\circ} \mathrm{C}$ for $10 \mathrm{~min}$.

\subsection{The competitive enzyme-linked immunosorbent assay} (ELISA)

In the study, both R5 ELISA and G12 ELISA were used to confirm the result of transamidation by mTG. The antigenicity of the 33mer peptide was estimated by RIDASCREEN® Gliadin Competitive kit (R-Biopharm, Darmstadt, Germany) and GlutenTox®ELISA Competitive (Biomedal, Seville, Spain). For RIDASCREEN ${ }^{\circledR}$ Gliadin Competitive Elisa (R5 ELISA), R5 monoclonal antibody (mAb) was used to recognize potential toxic peptides sequences of gliadins, such as the sequence of QQPFP and LQPFP. GlutenTox®ELISA Competitive (G12 ELISA) is an immunosorbent kit for the detection and quantification of the fraction of gliadin, secalin and barley, based on G12 mAb. G12 ELISA is specific for 33-mer peptide, which can detect QPQLPY, QPQLPF and so on.

In order to adjust the absorbance values into the range of the standard curve, samples were appropriately diluted in the buffer solution. The assays were carried out according to the manufacturer's instructions. The absorbance was then measured at $450 \mathrm{~nm}$ using a microplate reader (Bio-Rad, USA). The standard curve line of R5 ELISA was done using the cubic spline function and the standard curve line of G12 ELISA was performed by second-order polynomial regression.

\subsection{Statistical analysis}

Statistical analysis was performed using Lab Solution, ORIGIN 8.0 and RIDASOFTWin. The data is presented as mean \pm standard deviation (S.D.).

\section{Results}

\subsection{Modification of the 33-mer peptide by mTG}

3.1.1 RP-HPLC analysis. To investigate the effect of mTG on the 33-mer peptide, RP-HPLC was used to analyse the native peptide and its modified forms after MTG treatment combined with various acyl-acceptor substrates. Fig. 2 and Table 1 shows the data of the RP-HPLC analysis. All the following experiments were performed at least in triplicate.

The native 33-mer peptide is given in Fig. 2A. Fig. $2 \mathrm{~B}$ to $\mathrm{F}$ represent the 33-mer peptide treated with $\mathrm{mTG}$ and various acyl-acceptor substrates, while Fig. 2G shows the peptide treated without acyl-acceptor substrates. It was shown that new peaks were present after the enzyme catalysis, suggesting that retention time was different between the native peptide and its modified forms. Moreover, it was indicated that the retention time was reduced when the 33-mer peptide was crosslinked with LYS (Fig. 2B), LME (Fig. 2C), and HA (Fig. 2F). Meanwhile, the crosslinking of GEE (Fig. 2D) and 6-AA (Fig. 2E) had increased the retention time. The proportion of 33-mer peptide crosslinking with three LYS molecules can reach $90.26 \%$ (Table 1 ), while the ratio of 33-mer peptide crosslinking with three LME molecules, three GEE molecules, three 6-AA molecules and three HA molecules were 47.51, 42.12, 23.93 and $36.51 \%$, respectively. When the reaction mixture involved only mTG and 33-mer peptide, several new peaks appeared due to the deamidating activity of mTG (Fig. 2G). This experiment demonstrates the deamidation activity on the 33-mer peptide by mTG as such (Fig. 2G) and the mTG plus acyl-acceptor substrate-specific modifications in the other figures.

3.1.2 LC-ESI-MS analysis. To identify the effect of mTG treatment combined with acyl-acceptor substrates on the 33- 


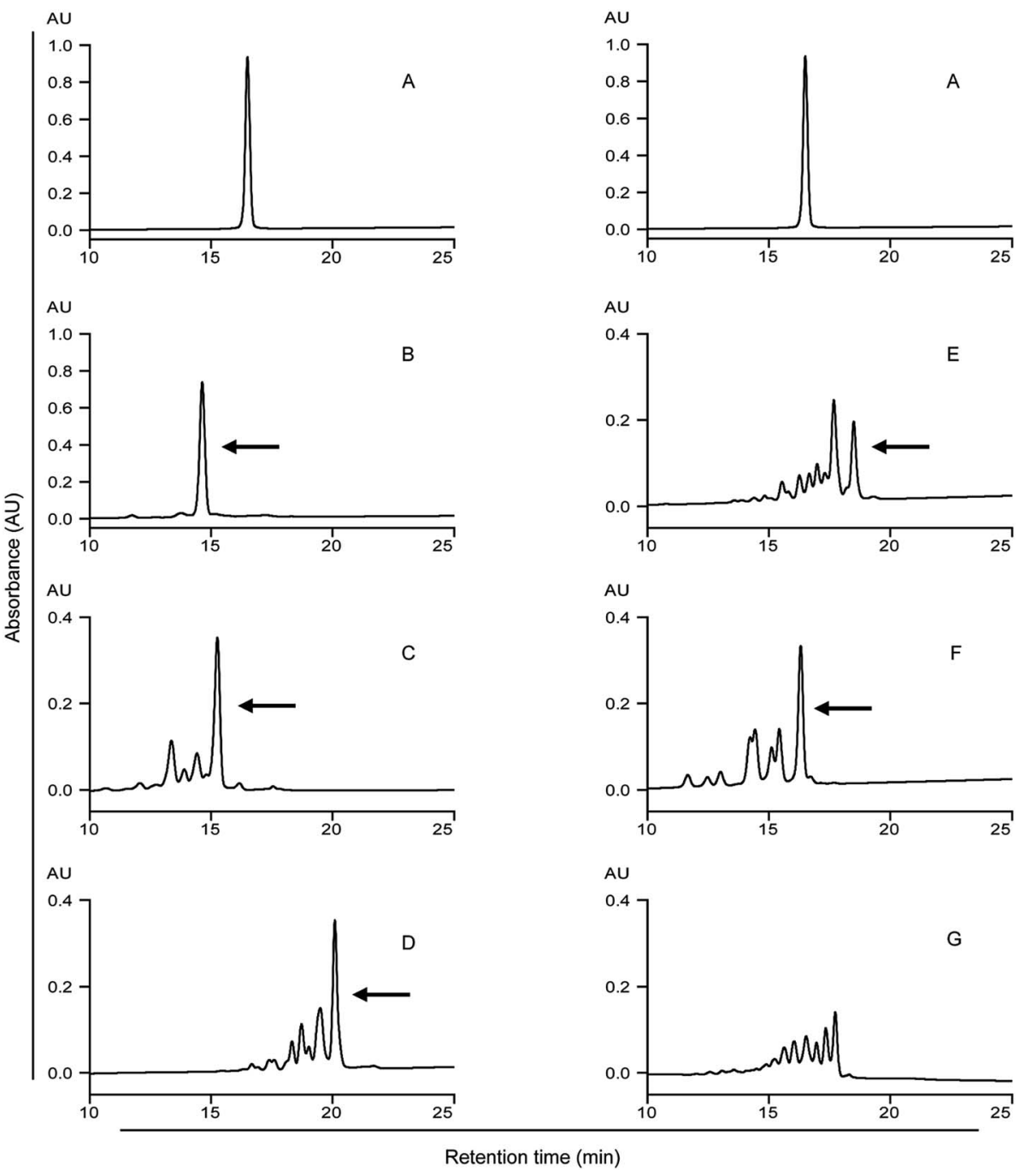

Fig. 2 HPLC analysis of the 33-mer peptide and its modified forms. Detecting wavelength is $220 \mathrm{~nm}$. (A) Native 33-mer peptide as control; (B) LYS/mTG; (C) LME/mTG; (D) GEE/mTG; (E) 6-AA/mTG; (F) HA/mTG; (G) 1 mg of 33-mer peptide and $0.5 \mathrm{U}$ mTG without acyl-acceptor substrates. Arrows indicate the 33-mer peptide crosslinked with three acyl-acceptor substrate molecules, which percentage is given in the Table 1. LYS: L-lysine; LME: L-lysine methyl ester; GEE: glycine ethyl ester; 6-AA: 6-aminocaproic acid; HA: hydroxylamine.

mer peptide, LC-ESI-MS was performed to analyse the native peptide and its modified forms. Fig. 1C shows the mechanism of mTG and Fig. 3 shows the data of the LC-ESI-MS analysis. All the following experiments were performed at least in duplicate.

In the LC-ESI-MS (Fig. 3A, Table 2), the $\mathrm{m} / \mathrm{z}$ value of the 33mer peptide was $978.8(4+)$, indicating that the measured molecular weight (MMW) was 3911.2 daltons. When the LYS molecules were crosslinked to the 33-mer peptide, a strong signal at $m / z$ value 860.7 (5+) was generated (Fig. 3B, Table 2), implying that the detected MW for the new product was 4298.5 daltons. By contrast, there was a shift of 387 daltons from the $\mathrm{m} /$ $z$ value $978.8(4+)(\mathrm{MMW}=3911.2$, theoretical average molecular weight $($ TAMW $)=3911.4)$ to $m / z$ value $860.7(5+)($ TAMW $=$ 4298.9 daltons), indicating the successful addition of three LYS molecules to the 33-mer peptide. However, the content of 33mer peptide crosslinking with a single LYS molecule or two LYS molecules was very low. Moreover, the native 33-mer peptide crosslinking with three LME molecules generated a strong signal at $m / z$ value $869.0(5+)$ (Fig. 3C, Table 2 ). The crosslinked 33 -mer peptide with one LME molecule $(\mathrm{m} / \mathrm{z}$ value $1014.9(4+))$ or two LME molecules $(\mathrm{m} / \mathrm{z}$ value $1050.5(4+))$ were also produced, but they had much lower abundance than the 33-mer peptide with three LME molecules (data not shown). Similarly, the 33-mer peptide crosslinking with three GEE molecules produced a strong signal at $m / z$ value $1043.2(4+)$ (Fig. 3D, Table 2 ), while the $m / z$ values $1000.2(4+)$ and $1021.8(4+)$ represented the crosslinking of the 33-mer peptide with one GEE molecule and two GEE molecules and the content of two products was low (data not shown). Regarding the 33-mer peptide with three 6-AA molecules, $m / z$ value $1064.2(4+)$ was observed (Fig. 3E, Table 2), while the signals of crosslinking with one 6-AA molecule or two 6-AA molecules were very weak (data not shown). In addition, the 33-mer peptide crosslinked with three HA molecules produced the $m / z$ value $990.7(4+)$ (Fig. 3F, Table 2), whereas the 

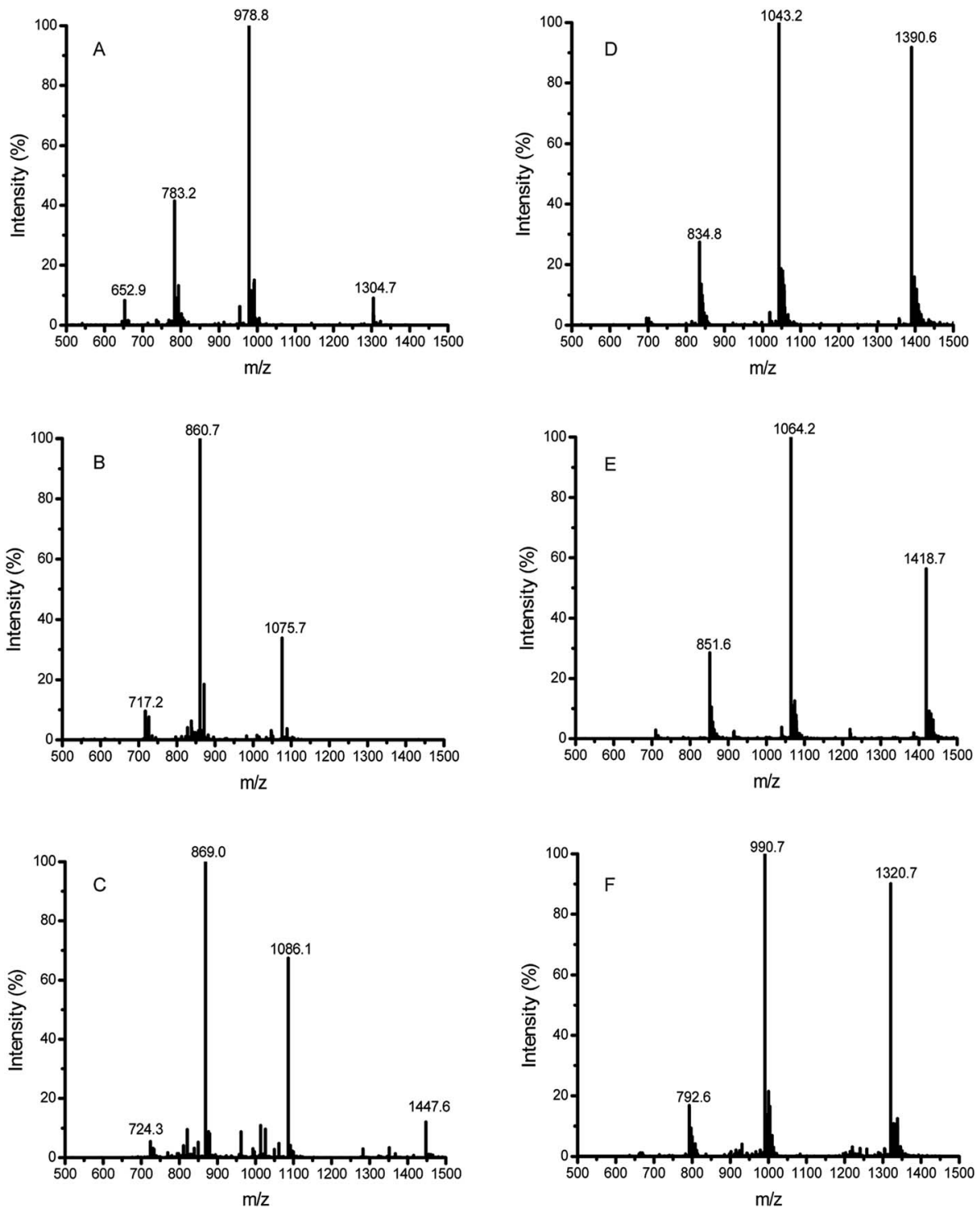

Fig. 3 HPLC-ESI-MS analysis of the 33-mer peptide and its transamidated forms. (A) Native 33-mer peptide as control; (B) addition of three LYS molecules to 33-mer peptide; (C) the 33-mer peptide crosslinked with three LME molecules; (D) the 33-mer peptide crosslinked with three GEE molecules; (E) the 33-mer peptide crosslinked with three 6-AA molecules; (F) the 33-mer peptide crosslinked with three HA molecules. LYS: Llysine; LME: L-lysine methyl ester; GEE: glycine ethyl ester; 6-AA: 6-aminocaproic acid; HA: hydroxylamine.

33-mer peptide with one HA or two HA molecules had weak signals (data not shown).

Regarding the reaction mixture with only $\mathrm{mTG}$ and the 33mer peptide, distinct $m / z$ values were observed (ESI Fig. 1B-E $\dagger$ ). According to the ESI Fig. 1B, $\dagger$ the $m / z$ values $979.4(4+)$ (MMW 3913.6) and 1305.7 (3+) (MMW 3914.1) arose, indicating a shift of 2-3 daltons from the native 33-mer peptide (MMW 3911.2). Therefore, it was concluded that 2-3 glutamine residues were deamidated to glutamic acid. Interestingly, incubation of 33mer peptide with mTG yielded different products, such as LQPFPQPQLPYPQPQLPYPQPQLPYPQPQPF (ESI Fig. 1C, $\dagger$ LQ from the native 33-mer peptide), QPFPQPQLPYPQPQLPYPQPQLPYPQPQPF (ESI Fig. 1D, $\dagger$ LQL from the native 33-mer peptide), and LQLQPFPQPQLPYPQPQLPYPQPQLPYPQP (ESI Fig. 1E, $\uparrow \mathrm{QPF}$ from the native 33-mer peptide).
Collecting the above results, all acyl-acceptor substrates in this work were active to crosslink with the 33-mer peptide by mTG and the 33-mer peptide has three crosslinking sites. Moreover, it demonstrates the deamidation activity and maybe degradation activity on the 33-mer peptide by mTG without acylacceptor substrates. However, it remains unclear which of the three glutamine residues were the modification sites and which site was modified first.

\subsection{Modification of the 33-mer peptide by enzymatic digestion}

To assess the digestibility of the modified peptides as produced after treatment with mTG plus the various acyl-acceptor substrates by pepsin, trypsin and chymotrypsin, we used HPLC to analyse the digested products. There were no 
Table 2 Identification of peptide and its main modified forms by HPLC-ESI-MS

\begin{tabular}{lcllll}
\hline Sample & $\begin{array}{l}\text { Precursor } \\
\text { ion }(m / z)\end{array}$ & Charge & MMW & TAMW & $\begin{array}{l}\text { No. of } \\
\text { added }\end{array}$ \\
\hline 33-mer & 978.8 & $4^{+}$ & 3911.2 & 3911.4 & \\
LYS & 808.9 & $5^{+}$ & 4039.5 & 4040.6 & $1^{a}$ \\
& 834.5 & $5+$ & 4167.5 & 4169.8 & $2^{a}$ \\
& 860.7 & $5+$ & 4298.5 & 4298.9 & 3 \\
LME & 1014.9 & $4+$ & 4055.6 & 4054.6 & 1 \\
& 1050.5 & $4+$ & 4198.0 & 4197.8 & 2 \\
GEE & 869.0 & $5+$ & 4340.0 & 4341.0 & 3 \\
& 1000.2 & $4+$ & 3996.8 & 3997.5 & 1 \\
& 1021.8 & $4+$ & 4083.2 & 4083.6 & 2 \\
6-AA & 1043.2 & $4+$ & 4168.8 & 4169.7 & 3 \\
& 1007.6 & $4+$ & 4026.4 & 4025.6 & $1^{a}$ \\
\multirow{4}{*}{ HA } & 1035.9 & $4+$ & 4139.6 & 4139.7 & 2 \\
& 1064.2 & $4+$ & 4252.8 & 4253.9 & 3 \\
& 1310.9 & $3+$ & 3929.7 & 3927.5 & $1^{a}$ \\
& 986.8 & $4+$ & 3943.2 & 3943.5 & $2^{a}$ \\
& 990.7 & $4+$ & 3958.8 & 3959.5 & 3
\end{tabular}

${ }^{a}$ Percentage < 5\%; MMW, measured molecular weight; TAMW, theoretical average molecular weight; L-lysine (LYS); L-lysine methyl ester (LME); glycine ethyl ester (GEE); 6-aminocaproic acid (6-AA); hydroxylamine (HA).

significant differences in digestibility between the modified peptides and the native 33-mer peptide (Fig. 4). After $1 \mathrm{~h}$ of in vitro gastric digestion, some new peaks appeared. As indicated in the Fig. $4 \mathrm{~A}_{2}-\mathrm{G}_{2}$, some peaks disappeared after $2 \mathrm{~h}$ of intestinal digestion. However, most of the initial peaks still remained. In summary, it is demonstrated that the enzymes from gastrointestinal tract failed to degrade the isopeptide.

\subsection{Inhibition of antigenicity by mTG}

3.3.1 R5 ELISA. To investigate whether the mTG modification can cause a decrease in the immunological properties of the 33-mer peptide, R5 ELISA immunoassay was used. According to the study of Kahlenberg et al., the sequences QQPFP, QQQFP, LQPFP, QLPFP and QLPYP can bind to R5 mAb as well as QLPYP that is repeated three times in the centre of 33-mer peptide and which can strongly stimulate the $\mathrm{T}$ cells in the celiac disease patients. ${ }^{24}$ In the ELISA immunoassays, the control group was regarded as $100 \%$ immunological activity, which contained the 33-mer peptide, inactivated enzyme and corresponding acyl-acceptor substrate. In comparison with the control group, the modified groups had low gliadin signal tested by the ELISA kits, indicating a decrease of binding activity of the epitopes in the 33-mer peptide to $\mathrm{R} 5 \mathrm{mAb}$ (Fig. 5A). The binding activity between R5 mAb and the 33-mer peptide crosslinking with LYS, LME, GEE, 6-AA and HA were decreased to $2.57,1.59,0.98,1.66$ and $5.17 \%$, respectively. After being deamidated by $\mathrm{mTG}$, the reactivity of the antibody declined to $67.70 \%$ of its initial level. To summarize, it was suggested that mTG can modify the 33-mer peptide, resulting in decreasing its antigenicity.

3.3.2 G12 ELISA. To further verify whether the mTG modification can cause a decrease in the immunological properties of the 33-mer peptide, G12 ELISA immunoassay was used.
Moreover, previous study indicated that the G12 mAb specifically recognized the sequence QPQLPY present in the 33-mer peptide of $\alpha 2$-gliadin. ${ }^{25}$ Regarding the G12 ELISA, when LYS, LME, GEE, 6-AA and HA were crosslinked to the 33-mer peptide, the antigenicity of the modified forms decreased to 26.21, 29.59, $29.96,30.26$ and $50.22 \%$ of its initial level, respectively (Fig. 5B). Whereas deamidation occurred in the mixture without acylacceptor substrates, the response to G12 mAb did not change. To sum up, it indicated that the transamidation activity of mTG in combination with the acyl-acceptor substrates greatly decreased the affinity of the 33-mer peptide binding to G12 $\mathrm{mAb}$.

\section{Discussion}

In this study, the obtained results show that the gliadin 33-mer peptide can be transamidated with mTG combined with different acyl-acceptor substrates. These results were in agreement with the previous study that the chemical and steric distinction of acyl-acceptor substrates gave rise to the different properties and reactivity. ${ }^{26}$ The most extensive modification of the 33-mer occurred when enzyme treatment was given using $1 \mathrm{mg}$ of 33-mer, $0.5 \mathrm{U}$ mTG and $0.05 \mathrm{mmol}$ LYS in a volume of 1 $\mathrm{mL}$ (Fig. 2B). That may be due to the reaction conditions being optimum for the reactivity of LYS. Interestingly, the crosslinking output level of the 33-mer peptide with three acylacceptor molecules was much higher than that of the 33-mer peptide with one acyl-acceptor molecular or two acyl-acceptor molecules in the same condition, demonstrating the 33-mer peptide has three transamidation sites. This work is in agreement with Mazzeo et al. that three Q sites were modified in the sequence of the 33-mer peptide (displayed in bold, LQLQPFPQPQLPYPQPQLPYPQPQLPYPQPQPF). ${ }^{20}$ Moreover, it has been shown that most of the modifications by mTG could be explained by mentioned patterns in the previous study that the Q is not a target of mTG in QP and QXXP sequences, while the $\mathrm{Q}$ is a target of mTG in the sequences QXP, QXX(FYWMLIV) and QXPF(YWMLIV). ${ }^{27}$ Actually, based on this mechanism, three $\mathrm{Q}$ modification sites were deduced in the 33-mer peptide, which are displayed in bold (33-mer: LQLQPFPQPQLPYPQP QLPYPQPQLPYPQPQPF). However, the occurrence of some other peaks in the HPLC spectrum remains unclear, except the peaks of the 33-mer peptide plus three acyl-acceptor substrates (Fig. 2). These may be due to the multifunction of mTG, including transamidation activity, deamidation activity and hydrolysis activity. Nevertheless, in view of our data, it has been shown that transamidation activity of $\mathrm{mTG}$ played a more important role compared to deamidation activity in the presence of acyl-acceptor substrates, which is consistent with the previous study that the transamidation rate was about 10 times higher than the deamidation rate in the presence of lysine. ${ }^{14}$

Furthermore, it indicated that all these peptides were degraded slightly after $1 \mathrm{~h}$ of in vitro gastric digestion and $2 \mathrm{~h}$ of in vitro intestinal digestion, which is consistent with a previous study in which the 33-mer peptide appeared to be highly resistant to digestion, even when exposed to enzymes of the brush-border membrane (BBM). ${ }^{6}$ After $1 \mathrm{~h}$ of gastric digestion, 


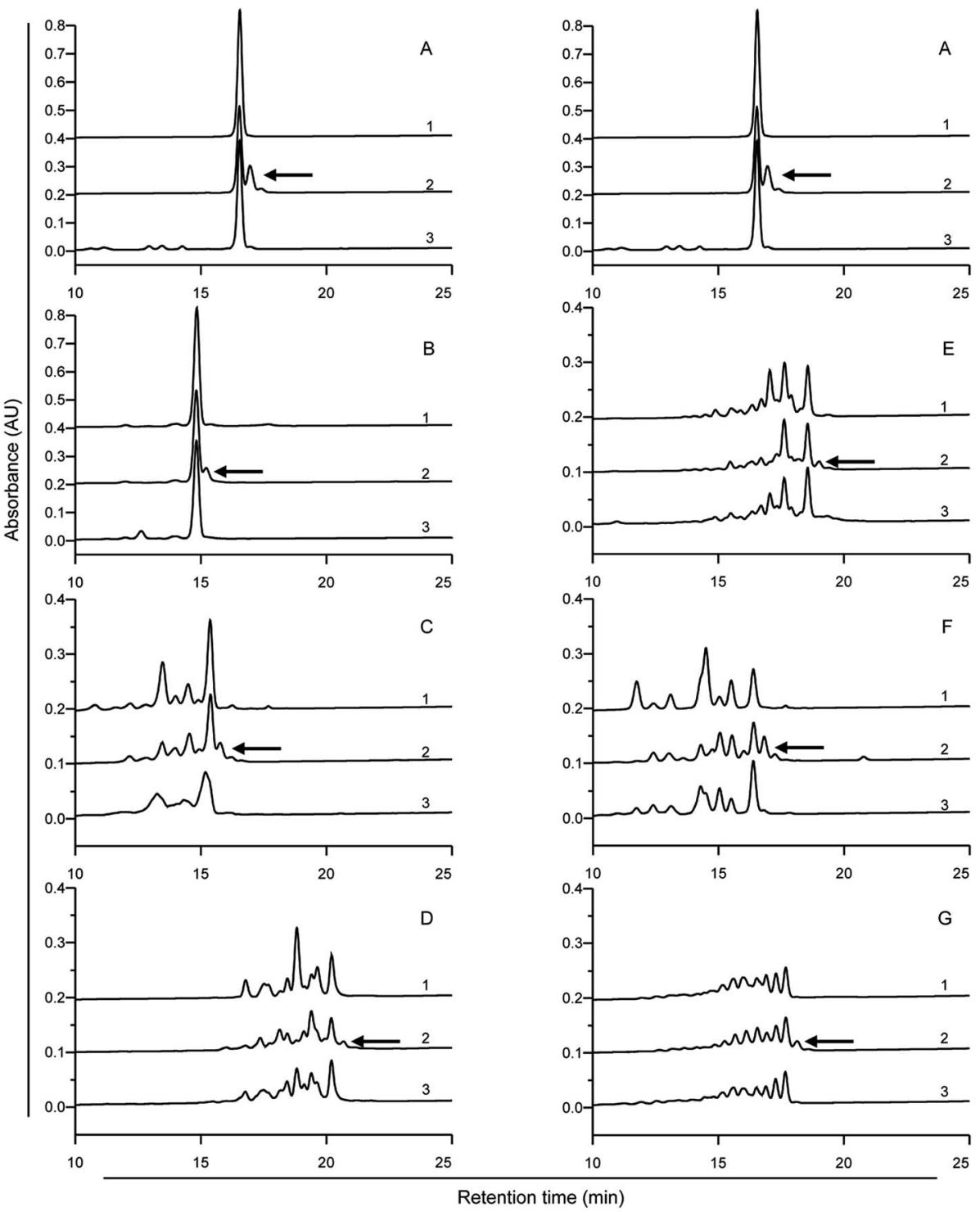

Fig. 4 HPLC analysis of the digestion profiles of the 33-mer peptide and its modified forms. The 33-mer peptide and its modified forms were incubated with simulated gastric fluid (SGF) at $\mathrm{pH} 2$ for $1 \mathrm{~h}$, followed by incubation with simulated intestinal fluid at pH 7 for $2 \mathrm{~h}$. Detecting wavelength is $220 \mathrm{~nm}$. (A) Native 33-mer peptide as control; (B) the crosslinked 33-mer peptide with LYS; (C) the crosslinked 33-mer peptide with LME; (D) the crosslinked 33-mer peptide with GEE; (E) the crosslinked 33-mer peptide with 6-AA; (F) the crosslinked 33-mer peptide with HA; (G) the deamidated 33-mer peptide. Arrows 1, 2 and 3 indicate the undigested peptides, peptides after gastric digestion and peptides after intestinal digestion, respectively. LYS: L-lysine; LME: L-lysine methyl ester; GEE: glycine ethyl ester; 6-AA: 6-aminocaproic acid; HA: hydroxylamine.

some new peaks appeared because amino-terminal leucine residue was cut by pepsin. ${ }^{6}$ However, most of the initial peaks still existed. Actually, these results were the same as found earlier showing that digestive enzymes were ineffective to degrade the gluten epitopes. ${ }^{28}$ In summary, it has been demonstrated that the enzymes from the gastrointestinal tract failed to degrade the isopeptide, which confirms that acylacceptor substrates remained linked to the peptide after in vitro digestion by pepsin and subsequently by pancreatin. ${ }^{29}$

The transamidation activity of mTG greatly decreased the affinity of the 33-mer peptide binding to R5 mAb. The sequence of LQPFP in the N-terminus of the 33-mer peptide can be recognized by the $\mathrm{R} 5 \mathrm{mAb}$, however, the sequence of QLPYP remains a weak reactivity to the antibody. ${ }^{24}$ The structural modification of three $\mathrm{Q}$ sites could be a reason for the reduction of $\mathrm{R} 5 \mathrm{mAb}$ recognition for the 33-mer peptide. ${ }^{24}$ Moreover, after being deamidated by $\mathrm{mTG}$, the reactivity of the antibody declined to $67.70 \%$ of its initial level. This is in accordance with the low antibody binding observed in previous studies showing that the substitution of glutamine with glutamic acid in the epitopes may decrease the recognition of R5 mAb.,24

Similarly, the transamidated forms had a low affinity to G12 $\mathrm{mAb}$ in comparison with the native 33-mer peptide. A previous study described that the G12 mAb strongly recognizes the sequence of QPQLPY, which is repeated three times in the 33peptide. ${ }^{25}$ Therefore, the decreased antigenicity was presented because acyl-acceptor substrates were crosslinked to Q site, which changed the structure of 33-mer peptide. However, there 

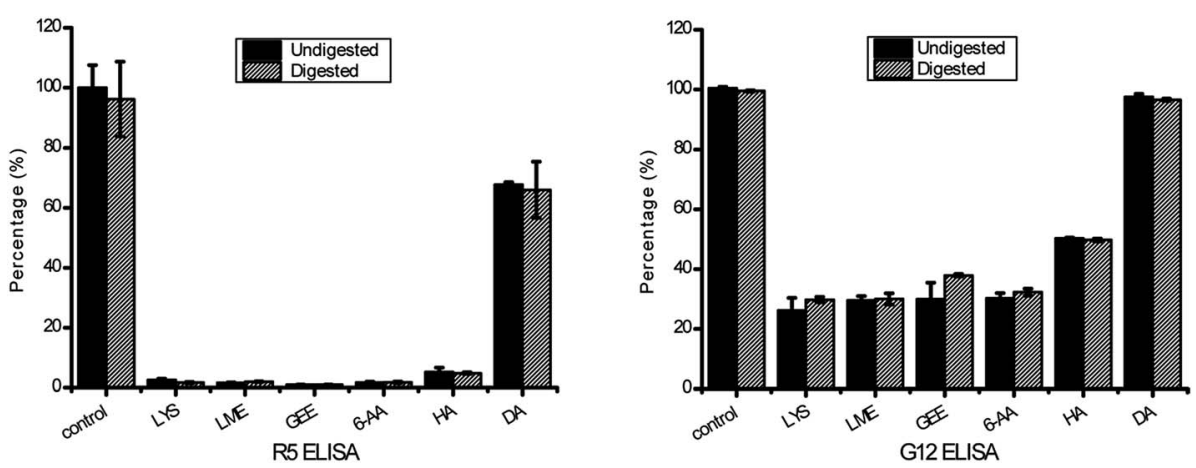

Fig. 5 Antigenicity of the 33-mer peptide and its modified forms tested by R5 competitive ELISA and G12 competitive ELISA. The untreated 33mer peptide contained $100 \%$ activity against R5 and G12 mAb as control group. The antigenicity of modified form is shown as percentage of the initial 33-mer peptide activity. Error bars indicated the standard deviation of at least three measurements. (A) The result detected by R5 competitive ELISA; (B) the result detected by G12 competitive ELISA. Columns represent the means \pm SD. mAb: monoclonal antibody; LYS: Llysine; LME: L-lysine methyl ester; GEE: glycine ethyl ester; 6-AA: 6-aminocaproic acid; HA: hydroxylamine; DA: deamidation.

was no obvious difference in the antigenicity between the untreated 33-mer peptide and the deamidated peptide, which was different from the previous study that the affinity of G12 mAb with the peptide QPQLPYPQP was much higher than for that of G12 mAb with the deamidated peptide QPELPYPQP. ${ }^{25}$ It may be due to incomplete deamidation of the 33-mer peptide in $2 \mathrm{~h}$ leading to the modified peptide have the similar immunological activity to the untreated peptide.

Additionally, the purity of 33-mer peptide crosslinked with three LYS molecules can reach to $90.26 \%$ (Table 1). When the 33-mer peptide was crosslinked with three LME molecules, three GEE molecules, three 6-AA molecules and three HA molecules, the purity was $47.51,42.12,23.93$ and $36.51 \%$, respectively. Nevertheless, there was no obvious difference between them in the binding activity to R5 and G12 mAb. It can be explained that 33-mer peptide crosslinked with three LYS molecules was more stable, while some of 33-mer peptide crosslinked with three LME molecules, three GEE molecules, three 6-AA molecules and three HA molecules was broken down because of the hydrolysis activity of mTG. For instance, aminoterminal LQL or carbon-terminal QPF was broken down by mTG from LQLQPF(PQPQLPY)3PQPQPF with three acyl-acceptor molecules as deamidation mixtures. Moreover, the $\mathrm{Q}$ sites were still crosslinked with these acyl-acceptor molecules. Therefore, most of transamidated forms had similar affinity R5 and G12 mAb.

Regarding the test of the digested product of the crosslinked 33-mer, it was concluded that there were only slight changes on the antigenicity according to the results of R5 ELISA and G12 ELISA and it is in agreement with the result of HPLC analysis that both native peptide and modified peptide could not be digested completely. Actually, it is also consistent with the observation that the L-lysine of $\varepsilon$-(y-L-glutamy1)-L-lysine was not released even after much prolonged incubation with the mucosal scraps. ${ }^{29}$ To summarize, it was suggested that mTG combined with acyl-acceptor substrates can modify the 33-mer peptide, resulting in decreasing its antigenicity.

In conclusion, it is suggested that a model 33-mer peptide related to CD can be modified in vitro by different acyl-acceptor substrates with mTG. After $2 \mathrm{~h}$ of transamidation, the antigenicity of the peptide decreased greatly compared to its initial level. The formation of isopeptide bonds probably prevents the presenting process by antigen presenting cells and the recognition by gluten-specific $\mathrm{T}$ cells. Moreover, this finding broadens the application of mTG to catalyse the gluten modification for reducing their immunogenicity in celiac disease. Additionally, in food processing, the crosslinking of various macromolecules to proteins strengthen food resistance to luminal protease, resulting in low digestibility and absorption. Nevertheless, the crosslinking of small acyl-acceptor molecules to proteins might exert only a weak impact on their digestibility and absorption, meanwhile inactivating their immunogenicity. Strikingly, the small acyl-acceptor molecules would be costeffective for applications of the food industry, in comparison with the macromolecules. Overall, the results might provide a new sight to develop new types of food with better nutritional, structural characteristics and being safe for people suffering from celiac disease.

\section{Conflict of interest}

The authors declare that there are no conflicts of interest.

\section{Acknowledgements}

The work was supported by the International Science \& Technology Cooperation Program of China (No. 2013DFG31380), National High Technology Research and Development Program of China (863 Program; No. 2013AA102205), and the Research Program of State Key Laboratory of Food Science and Technology (No. SKLF-ZZA-201612).

\section{References}

1 J. F. Ludvigsson, D. A. Leffler, J. C. Bai, F. Biagi, A. Fasano, P. H. Green, M. Hadjivassiliou, K. Kaukinen, C. P. Kelly and J. N. Leonard, Gut, 2013, 62, 43-52.

2 V. Abadie, L. M. Sollid, L. B. Barreiro and B. Jabri, Annu. Rev. Immunol., 2011, 29, 493-525. 
3 C. Kristiansen, L. Madsen, L. Fugger, H. Scott, O. Norén, P. Roepstorff, K. E. Lundin, H. Sjöström and L. M. Sollid, Nat. Med., 1998, 4, 713-717.

4 Y. van de Wal, Y. Kooy, P. van Veelen, S. Peña, L. Mearin, G. Papadopoulos and F. Koning, J. Immunol., 1998, 161, 1585-1588.

5 S. Tollefsen, H. Arentz-Hansen, B. Fleckenstein, Ø. Molberg, M. Ráki, W. W. Kwok, G. Jung, K. E. Lundin and L. M. Sollid, J. Clin. Invest., 2006, 116, 2226.

6 L. Shan, Ø. Molberg, I. Parrot, F. Hausch, F. Filiz, G. M. Gray, L. M. Sollid and C. Khosla, Science, 2002, 297, 2275-2279.

7 J. Van de Kamer and H. Weijers, Acta Paediatr., 1955, 44, 465469.

8 S.-W. Qiao, E. Bergseng, Ø. Molberg, J. Xia, B. Fleckenstein, C. Khosla and L. M. Sollid, J. Immunol., 2004, 173, 1757-1762.

9 B. Meresse, G. Malamut and N. Cerf-Bensussan, Immunity, 2012, 36, 907-919.

10 V. Capriles and J. Arêas, Food Funct., 2016, 7, 1266-1272.

11 M. Bustos, G. Perez and A. Leon, RSC Adv., 2015, 5, 3078030792.

12 F. Cabrera-Chávez, A. R. Islas-Rubio, O. Rouzaud-Sández, N. Sotelo-Cruz and A. M. C. de la Barca, J. Cereal Sci., 2010, 52, 310-313.

13 J. Folk and S. I. Chung, Methods Enzymol., 1985, 113, 358-375. 14 A. Heil, J. Ohsam, C. Büchold, R. Pasternack, K. Yokoyama, Y. Kumazawa and M. Hils, J. Cereal Sci., 2016, 70, 47-56.

15 N. G. Heredia-Sandoval, A. R. Islas-Rubio, F. Cabrera-Chávez and A. M. C. de la Barca, Food Funct., 2014, 5, 1813-1818.

16 C. Gianfrani, R. A. Siciliano, A. M. Facchiano, A. Camarca, M. F. Mazzeo, S. Costantini, V. M. Salvati, F. Maurano, G. Mazzarella and G. Iaquinto, Gastroenterology, 2007, 133, 780-789.

17 L. Elli, L. Roncoroni, M. Hils, R. Pasternack, D. Barisani, C. Terrani, V. Vaira, S. Ferrero and M. T. Bardella, Hum. Immunol., 2012, 73, 992-997.
18 G. Mazzarella, V. M. Salvati, G. Iaquinto, R. Stefanile, F. Capobianco, D. Luongo, P. Bergamo, F. Maurano, N. Giardullo and B. Malamisura, Clin. Dev. Immunol., 2012, 2012, 329150.

19 E. Lombardi, P. Bergamo, F. Maurano, G. Bozzella, D. Luongo, G. Mazzarella, V. R. Aufiero, G. Iaquinto and M. Rossi, J. Leukocyte Biol., 2013, 93, 479-488.

20 M. F. Mazzeo, R. Bonavita, F. Maurano, P. Bergamo, R. A. Siciliano and M. Rossi, Biochim. Biophys. Acta, Gen. Subj., 2013, 1830, 5166-5174.

21 C.-H. Tang, X. Sun, S.-W. Yin and C.-Y. Ma, Food Res. Int., 2008, 41, 941-947.

22 J. Folk and P. Cole, Biochim. Biophys. Acta, Enzymol. Biol. Oxid., 1966, 122, 244-264.

23 L. Shan, S.-W. Qiao, H. Arentz-Hansen, Ø. Molberg, G. M. Gray, L. M. Sollid and C. Khosla, J. Proteome Res., 2005, 4, 1732-1741.

24 F. Kahlenberg, D. Sanchez, I. Lachmann, L. Tuckova, H. Tlaskalova, E. Méndez and T. Mothes, Eur. Food Res. Technol., 2006, 222, 78-82.

25 B. Morón, M. T. Bethune, I. Comino, H. Manyani, M. Ferragud, M. C. López, Á. Cebolla, C. Khosla and C. Sousa, PLoS One, 2008, 3, e2294.

26 M. T. Gundersen, J. W. Keillor and J. N. Pelletier, Appl. Microbiol. Biotechnol., 2014, 98, 219-230.

27 E. Dekking, P. Van Veelen, A. De Ru, E. Kooy-Winkelaar, T. Gröneveld, W. Nieuwenhuizen and F. Koning, J. Cereal Sci., 2008, 47, 339-346.

28 G. Janssen, C. Christis, Y. Kooy-Winkelaar, L. Edens, D. Smith, P. van Veelen and F. Koning, PLoS One, 2015, 10, e0128065.

29 K. Iwami and K. Yasumoto, J. Sci. Food Agric., 1986, 37, 495503. 